\title{
Application of Analytic Hierarchy Process in Underwater Robot Competition
}

\author{
Yueyi Chen ${ }^{1, a}$, Yansong Deng ${ }^{1, b, *}$ \\ ${ }^{1}$ Institute of Electrical and Information Engineering, Southwest Minzu University, West Terminal Street, \\ Chengdu, China \\ ${ }^{2}$ Department of Communication Engineering, Southwest Minzu University, West Terminal Street, \\ Chengdu, China \\ a997822116@qq.com, b342547288@qq.com, \\ *corresponding author
}

Keywords: Underwater robot; Mathematical model; Analytic Hierarchy Process; Underwater; Wrestling Match

\begin{abstract}
The analytic hierarchy process in mathematical modelling can be applied to numerous fields, even in the underwater competition. In this paper, we took the one of the related competitions, underwater wrestling match for example, when the robot pushes the point in different areas, the force exerted on the float by robot is different, and the speed, direction, reaction time of the robot as well as the distance from the specified point will have influences on the result of the game, too. We divided the float into four areas, using AHP to study the weight of each area, and finally determine the optional strategy. Having been verified, the experimental results are consistent with the theory.
\end{abstract}

\section{Introduction}

With the rapid developments of biological and engineering technology sciences, the boom of underwater robot such as robot fish has been set off in the recent years. Having experienced a long process of the adaption to the environment as well as the procedure of natural selection, fishes have evolved a remarkable ability in underwater movements. Some promote high swimming efficiency, motility, agility and concealment with the propulsion of caudal fin, which cannot be matched by the traditional propeller-propelled shipping tools. Therefore, numerous domestic and foreign research institutions go into underwater robot which is an emerging frontier technology, the relevant competition also came into being. It is foreseeable that in the near future, underwater robot can operate automatically in the complex environment and plays a significant role in the fields of marine environmental testing, submarine exploration, marine rescue, military reconnaissance and so on. Related competitions will get more attention and participation. In this paper, we established a mathematical model based on the impacts of underwater robots' mechanical structure and motion parameters on competition, and used analytic hierarchy process to get the optimal strategy.

\section{Underwater Wrestling Match}

\subsection{Rules of the competition}

Each team sends one underwater robot to participate in the competition. The pool is divided into two areas by a line which connects two centre points of the two long sides. The size of each area is $1.5 \mathrm{~m} \times 2 \mathrm{~m}$. At the beginning of the competition, the referee puts the float in the water, requiring the centre of the float and the centre of the pool remain stationary, then, place the robots of both side inside the floating object as is shown in Fig. 1. When the competition starts, fish A will push the float to the left, B to the right. If the robot in team A takes the lead in pushing the floating 
objects completely into the left area within 1 minute, the team $\mathrm{A}$ wins, and the game ends; Otherwise, if the robot of team B pushes the float into the right area firstly, the game will end with team B wining. If neither of the two cases above is present, we will compare two areas of the float both sides occupied in the first one minute, the larger side will win, and the game ends. If two areas are equal at this time, the game will be directly put into the overtime, the game won't end until the areas of the float occupied by two sides are different, and the larger side will win ${ }^{[1]}$.

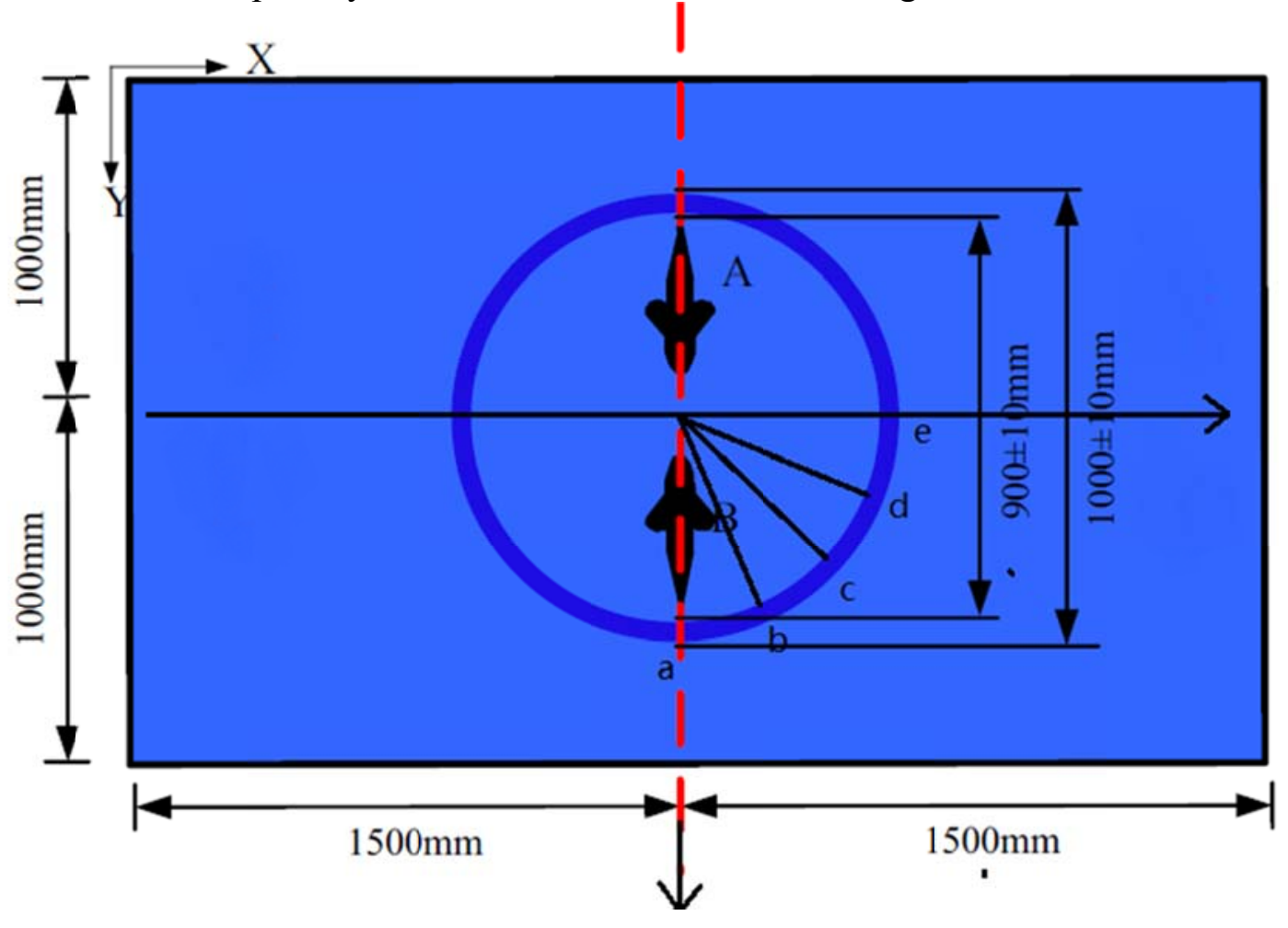

Fig. 1 Underwater Wrestling Match

\subsection{Analysis of strategies}

If we want the underwater robot to push float to the target area in the shortest possible time and get victory of the game, it is necessary for us to run ahead of the opponent in time of reaching the float. In this case, we assume that the opponent starts from the starting point and begins to push the float at the position as shown in the Fig. 1. If we want to be ahead of the opponent, our robot must reach the corresponding point before the opponent touches the float. According to the previous experiences of competitions, we can divide the game time by 4 times, and the time we are going to research is the first 15 seconds at the start of the race. Assuming that our robot starts from the starting point, the intervals of the arrival point can be divided into [a, b], [b, c], [c, d], [d, e], we name the four areas as Area 1, Area 2, Area 3, Area 4. The points on each different interval have the advantages and disadvantages of velocity, direction, distance and the reaction time, for example, it takes the shortest for the robot to arrive at the point on the Area 1, but the component of the force which the robot exerts on the float in the horizontal direction is the smallest. Under the condition of exerting the same force on the point in the Area 4, the force on the float is the largest, while it is time-consuming. So we have to combine the characteristics of each interval to find a best point and determine the optional strategy.

\section{The Mechanical Structure and Movement Patterns of the Underwater Robot}

\subsection{The mechanical structure of the underwater robot}

In the process of moving, the first $2 / 3$ part of its body is of great stiffness, we take robot fish for example, almost maintaining rigidity. The body wave amplitude is mainly in the third part of the 
body and reaches the maximum at the end of the tail. $90 \%$ of the propulsion force is produced by the caudal fin movement, so the movement of the caudal fin is the key to our study ${ }^{[2]}$. While the caudal fin movement is the composition of translational motion and swing motion. The internal structure of the robot is shown in the Fig. 2, the shape of the robot is spindle-shaped, such shape design can keep the laminar state of the boundary layer without causing the flow separation, the movement of the caudal fins is the motion synthesis of two rotating joints, and the movements of the two joints satisfies a certain phase following relationship.
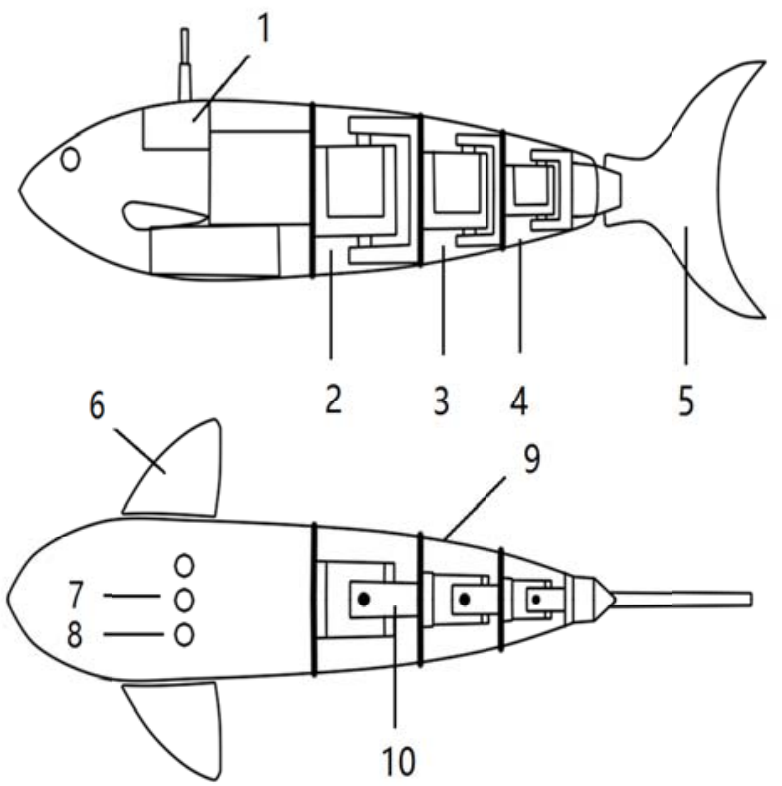

1 Communication module 2 Joint one 3 Joint two
4 Joint three 5 Caudal fin 6 Pectoral fin 7 Antennae
8 Charging plug 9 Waterproof skin 10 Aluminium alloy frame

Fig. 2 The Mechanical Structure of the Underwater Robot

\subsection{The movement patterns of the underwater robot}

The movements of the robot fish mainly include following forms:

1) Linear motion: tail and caudal fins swing according to the axis of the robot body as the axis of symmetry, in a swing cycle, the deflection force of both sides offsets each other, the robot moves along a straight line.

2) Steering movement: basic turning modes include moving forward turn, inertia forward turn and still turn.

3) Ascending and diving movement: During the procedure of moving forward, the robot changes the angle that pectoral fin hits the water, achieving ascending and diving movement via the lift force produced by pectoral fins ${ }^{[3]}$

In summary, the mechanical structure of the robot in the wrestling competition mainly affects the results of the game from two aspects: the distance and the direction.

\section{Establish Mathematical Model of Robot Path via AHP}

\subsection{Introduction to Analytic Hierarchy Process}

Analytic Hierarchy Process (AHP) is an easy way to make decisions about some of the complex and vague problems, and it is particularly appropriate for problems which are difficult to fully quantify ${ }^{[4]}$.It is a simple, flexible and practical multi-criteria decision-making method put forward by Professor T. L. Saaty, an American operational scientist, in the early 1970s. 


\subsection{Establishment and Solution to Mathematical Model of Robot Path}

In order to find the optional strategy, we set the Goal (represented by A )as the best area where the optional point lies; the main factors affecting the best point include speed, direction (angle), distance and reaction time, we regard these factors as the Criterion (represented by B); four areas as the Alternative(represented by C).

Goal

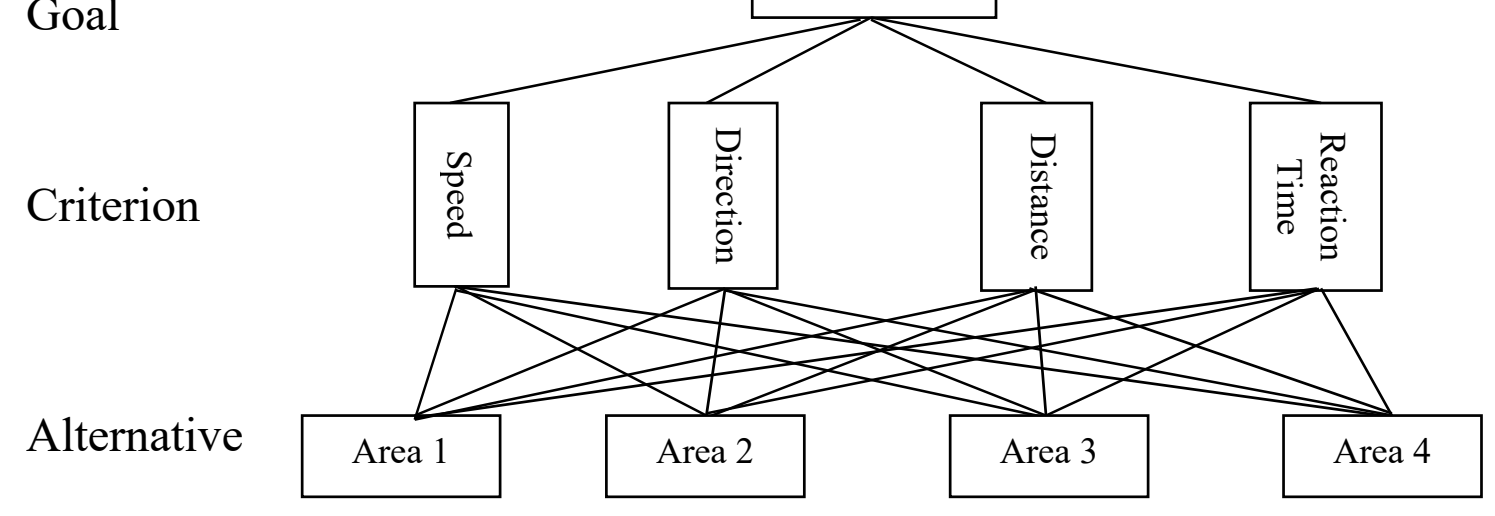

Numerical scales of factor contribution according to experience and judgment ${ }^{[5]}$ :

Table 1 The Meaning of the Numerical Scales

\begin{tabular}{|c|c|}
\hline Numerical scales $\mathrm{a}_{\mathrm{i} j}$ & The meaning of the numerical scales \\
\hline 1 & Factors $\mathrm{i}$ and $\mathrm{j}$ contribute equally to the objective \\
\hline 3 & Factors $\mathrm{i}$ is favored slightly over factor $\mathrm{j}$ \\
\hline 5 & Factors $\mathrm{i}$ is favored strongly over factor $\mathrm{j}$ \\
\hline 9 & Factors $\mathrm{i}$ is favored very strongly over factor $\mathrm{j}$ \\
\hline $2,6,8$ & Factors $\mathrm{i}$ is extremely important over factor $\mathrm{j}$ \\
\hline $\begin{array}{c}\text { The intermediate values reflect intermediate } \\
\text { position of importance }\end{array}$ \\
\hline $\begin{array}{c}\text { Reciprocals(i.e.1,1/3,1 } \\
15 \ldots 1 / 9)\end{array}$ & $\begin{array}{r}\text { The reciprocal number reflects the reverse } \\
\text { comparison positions of above }\end{array}$ \\
\hline
\end{tabular}

After reviewing a large number of references and on the basis of previous experience over the years, assuming that the midpoint of each area represents the point where the robot pushes the float, we can get:

The comparison matrix of Criterion is shown in Table 2. 
Table 2 Criterion Comparison Matrix

\begin{tabular}{|l|lccc|}
\hline A & B1 & B2 & B3 & B4 \\
\hline B1 & 1 & $1 / 2$ & $1 / 7$ & $1 / 9$ \\
B2 & 2 & 1 & $1 / 5$ & $1 / 8$ \\
B3 & 7 & 5 & 1 & $1 / 2$ \\
B4 & 9 & 8 & 2 & 1 \\
\hline
\end{tabular}

The comparison matrix of Alternative is shown in Table 3.

Table 3Alternative Comparison Matrix

\begin{tabular}{|ccccc|ccccc|}
\hline B1 & C1 & C2 & C3 & C4 & B2 & C1 & C2 & C3 & C4 \\
C1 & 1 & 3 & 7 & 9 & C1 & 1 & $1 / 3$ & $1 / 4$ & $1 / 6$ \\
C2 & $1 / 3$ & 1 & 3 & 5 & C2 & 3 & 1 & $1 / 3$ & $1 / 5$ \\
C3 & $1 / 7$ & $1 / 3$ & 1 & 3 & C3 & 4 & 3 & 1 & $1 / 3$ \\
C4 & $1 / 9$ & $1 / 5$ & $1 / 3$ & 1 & $\mathbf{C 4}$ & 6 & 5 & 3 & 1 \\
\hline B3 & $\mathbf{C 1}$ & $\mathbf{C 2}$ & $\mathbf{C 3}$ & $\mathbf{C 4}$ & $\mathbf{B} 4$ & $\mathbf{C 1}$ & $\mathbf{C 2}$ & $\mathbf{C 3}$ & $\mathbf{C 4}$ \\
C1 & 1 & $1 / 3$ & $1 / 5$ & $1 / 7$ & $\mathbf{C 1}$ & 1 & 1 & 2 & 3 \\
$\mathbf{C 2}$ & 3 & 1 & $1 / 3$ & $1 / 5$ & $\mathbf{C 2}$ & 1 & 1 & 1 & 2 \\
C3 & 5 & 3 & 1 & $1 / 3$ & $\mathbf{C 3}$ & $1 / 2$ & 1 & 1 & 1 \\
C4 & 7 & 5 & 3 & 1 & $\mathbf{C 4}$ & $1 / 3$ & $1 / 2$ & 1 & 1 \\
\hline
\end{tabular}

The procedure of the consistency check of the comparison matrix is shown below:

1) Calculate the consistency index (CI)

$$
\mathrm{CI}=\frac{\lambda_{\max }-n}{n-1}
$$

2) Find the corresponding average random consistency index (RI) ${ }^{[6]}$.The values of RI are given for $\mathrm{n}=1, \mathrm{~L}, 9$, by Saaty, which are shown in Table 4 .

Table 4 The Value of RI

\begin{tabular}{|c|ccccccccc|}
\hline $\mathbf{N}$ & $\mathbf{1}$ & $\mathbf{2}$ & $\mathbf{3}$ & $\mathbf{4}$ & $\mathbf{5}$ & $\mathbf{6}$ & $\mathbf{7}$ & $\mathbf{8}$ & $\mathbf{9}$ \\
\hline $\mathbf{R I}$ & 0 & 0 & 0.58 & 0.90 & 1.12 & 1.24 & 1.32 & 1.41 & 1.45 \\
\hline
\end{tabular}

The value of RI is obtained by constructing 500 sample matrices via random method: we construct reciprocal matrix with numbers which are extracted from 1 to 9 and their reciprocal randomly to obtain the mean value of the largest eigenvalue $\lambda_{\max }^{\prime}$ and we define:

$$
\mathrm{RI}=\frac{\lambda_{\max }^{\prime}-n}{n-1}
$$


3) Calculate consistency ratio

$$
\mathrm{CR}=\frac{C I}{R I}
$$

When $\mathrm{CR}<0.10$, it is considered that the consistency of the comparison matrix is acceptable, otherwise the comparison matrix should be corrected properly.

The result of the matrix solution is shown in Table 5:

Table 5 The Values of CR in Consistency Check

\begin{tabular}{|c|c|c|c|c|}
\hline $\mathbf{B}$ & $\mathbf{B}_{\mathbf{1}}$ & $\mathbf{B}_{\mathbf{2}}$ & $\mathbf{B}_{\mathbf{3}}$ & $\mathbf{B}_{\mathbf{4}}$ \\
\hline $\mathbf{C R}$ & 0.0325 & 0.0545 & 0.0433 & 0.0301 \\
\hline
\end{tabular}

It can be obtained from the table that, the value of CR from each Alternative is $<0 .{ }^{[7]}$, so they all passed the consistency test.

Having been calculated by Matlab, the total taxis of hierarchy is shown in Table 6 .

Table 6 The Total Taxis of Hierarchy

\begin{tabular}{|l|c|cccc|c|}
\hline \multicolumn{2}{|c|}{ Criterion } & Speed & Direction & Distance & $\begin{array}{c}\text { Reaction } \\
\text { time }\end{array}$ & $\begin{array}{c}\text { Total } \\
\text { sequencing } \\
\text { weights }\end{array}$ \\
\hline \multicolumn{2}{|c|}{$\begin{array}{c}\text { The weighs of } \\
\text { Criterion }\end{array}$} & 0.0478 & 0.0754 & 0.3244 & 0.5525 & \\
\hline $\begin{array}{l}\text { The single } \\
\text { ordering }\end{array}$ & Area1 & 0.6073 & 0.0629 & 0.0553 & 0.3677 & 0.1071 \\
$\begin{array}{l}\text { weight } \\
\text { values of } \\
\text { Alternative }\end{array}$ & Area2 & 0.2427 & 0.1226 & 0.1175 & 0.2819 & 0.4039 \\
& Area3 & 0.1015 & 0.2559 & 0.2622 & 0.2000 & 0.4087 \\
\end{tabular}

According to the weights of the total taxis of hierarchy, the optimal target point falls in Area 3.

\subsection{Result Validation}

In the robot program, we appointed the area where the target point lied is from the Area one to four. When the target point is in the Area 3, the speed that underwater robot pushes the float to the right area is the fastest, and it is the most advantageous way for us to obtain excellent results of the game.

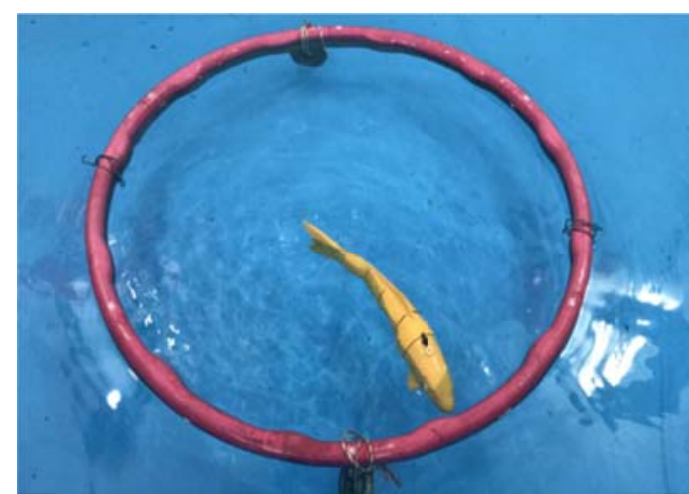

Fig.3 Robot pushing point in Areal

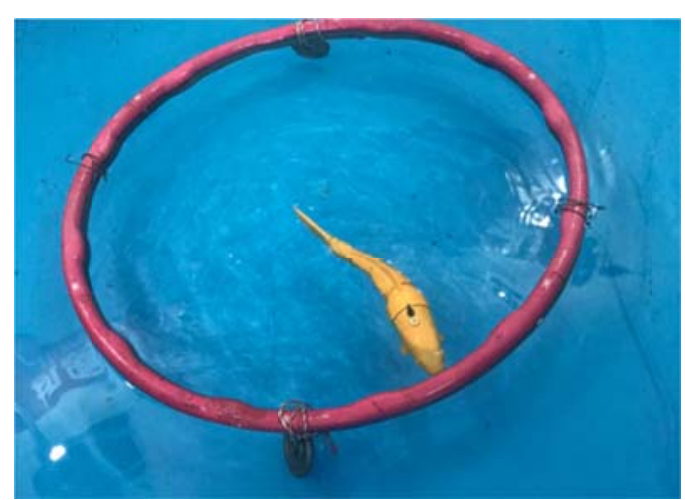

Fig.4 Robot pushing point in Area2 


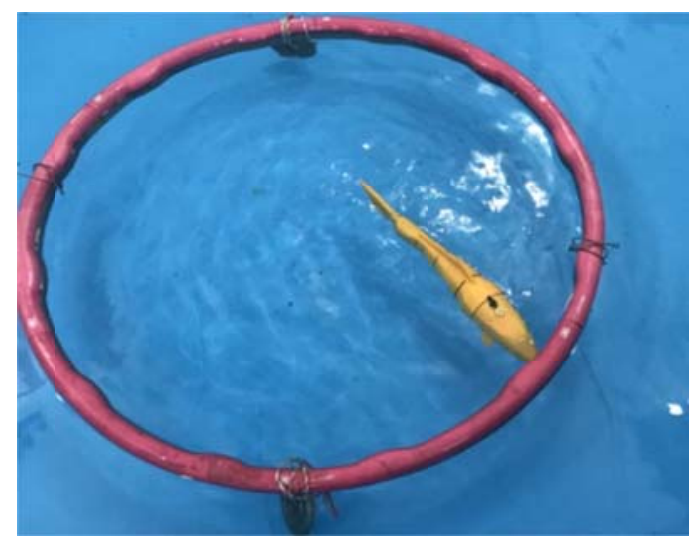

Fig.5 Robot pushing point in Area3

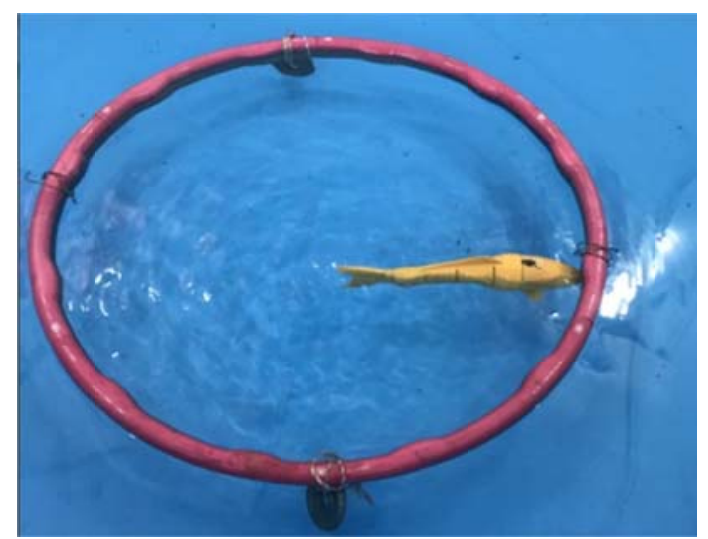

Fig.6 Robot pushing point in Area4

\section{Conclusion}

Having established mathematical model, using analytic hierarchy process to consider the impact of various factors on the performance of underwater robot performance comprehensively, we calculated the various weights rationally, analysed and determined the optional point scientifically, which is very helpful for getting excellent results in the wrestling match.

\section{Acknowledgements}

This work was partially supported by National Nature Science Foundation (61673016), SWUN Construction Projects for Graduate Degree Programs (2017XWD-S0805), Advance Research Program of Electronic Science and Technology National Program (2017YYGZS 16) and Sichuan Youth Science and technology innovation research team (2017TD0028), also was financially supported by students innovation training project of SWUN (201710656006).

\section{References}

[1]Guangming Xie. Introduction of robot water polo competition project [M]. Beijing: Peking University Institute of Technology, 2009: 1-5.

[2]Michael Sfakiotakis, Lane D M, Bruce J , et al .Review of Fish Swimming Modes for Aquatic Locomotion [ J ] . IEEE Journal of Oceanic Engineering, 1999, 34 (2): 237252.

[3]Qingxin Meng, Xinghua Zhu, Liquan Wang, Ping Li. Design and Realization of Bionic Machine Fish Control System Based on AVR Single Chip Microcomputer. Harbin Engineering University, $1004373 X$ ( 2005) 1210804.

[4]Saaty T. The analytic hierarchy process. New York: McGraw-Hill; 1980.

[5]Yingluo Wang. Systems engineering [M].Edition two.Beijing: Machinery Industry Press, 2003,130-140.

[6]Augusto, S.C., Mol, A.C.A., Jorge, C.A.F., 2007. Use of virtual reality to estimate ra- diation dose rates in nuclear plants. In: International Atlantic Conference-INAC 2007, Santos, SP, Brazil.

[7]Saaty, T.L., 1994. How to make a decision: the analytic hierarchy process.Interfaces24 (6), 19e43. 